PROCEEDINGS OF THE AMERICAN MATHEMATICAL SOCIETY

Volume 127, Number 3, March 1999, Pages 771-778

S 0002-9939(99)04791-7

\title{
ON THE QUINTUPLE PRODUCT IDENTITY
}

\author{
HERSHEL M. FARKAS AND IRWIN KRA
}

(Communicated by Dennis A. Hejhal)

\begin{abstract}
In this note we present a new proof of the quintuple product identity which is based on our study of $k^{t h}$ order theta functions with characteristics and the identities they satisfy. In this context the quintuple product identity is another example of an identity which when phrased in terms of theta functions, rather than infinite products and sums, has a simpler form and is much less mysterious.
\end{abstract}

\section{INTRODUCTION}

One of the important combinatorial identities which has received much attention is the celebrated quintuple product identity (QPI). It is in a certain sense, a generalization of the Jacobi triple product identity. While many proofs of of QPI are available, [9], [8], [3] ${ }^{1}$, it seems desirable to give a proof which in a sense explains the identity and relates it to a circle of ideas which have been quite useful in studying modular curves and their mappings into projective space of relatively small dimension. This material can be found in [5] and a survey of results can be found in [2].

A new proof of QPI was recently given by K. Alladi [1] which used the splitting of certain series into even and odd parts. Our proof, though related to this idea, is quite different in that it uses the decomposition of the vector space of theta functions of order $k$ with characteristic $\left[\begin{array}{l}1 \\ 1\end{array}\right]$ into subspaces of even and odd functions. It is the even functions, more precisely their values at the origin, which play a crucial role in other areas. It is these functions that in fact give and explain the QPI. As in almost all proofs, Euler's pentagonal theorem and the Jacobi triple product identity come into play, but now in the context of theta functions where they are more understandable (to us, at least). Finally we remark that QPI is another example of an identity that has a much simpler form when written in terms of theta functions with rational characteristics rather than in some other way.

Received by the editors June 17, 1997.

1991 Mathematics Subject Classification. Primary 30F30, 11F03; Secondary 30B99, 14H05, 05A30.

Key words and phrases. $q$-series, $k^{\text {th }}$ order theta functions with characteristics, partitions of integers, Jacobi triple product, Euler pentagonal number theorem.

The second author's research was supported in part by NSF Grant DMS 9500557. The first author's research was supported in part by the Gabriella and Paul Rosenbaum Foundation and the Edmund Landau Center for Research in Mathematical Analysis sponsored by the Minerva Foundation Germany. Both authors were supported in part by a US-Israel BSF Grant 95-348.

${ }^{1}$ This paper contains remarks on the interesting history behind the identity. 
From the definition of the theta functions given in $\S 2$, we see that for an odd positive integer $k$ and arbitrary integer $l$,

$$
\theta\left[\begin{array}{c}
\frac{2 l+1}{k} \\
1
\end{array}\right](k(\zeta+1), k \tau)=-\theta\left[\begin{array}{c}
\frac{2 l+1}{k} \\
1
\end{array}\right](k \zeta, k \tau)
$$

and

$$
\theta\left[\begin{array}{c}
\frac{2 l+1}{k} \\
1
\end{array}\right](k(\zeta+\tau), k \tau)=-\exp \left(-2 \pi \imath\left[k \zeta+k \frac{\tau}{2}\right]\right) \theta\left[\begin{array}{c}
\frac{2 l+1}{k} \\
1
\end{array}\right](k \zeta, k \tau) .
$$

Hence these are $k^{t h}$ order theta functions with characteristic $\left[\begin{array}{l}1 \\ 1\end{array}\right]$. The space of $k^{t h}$ order theta functions with characteristic $\left[\begin{array}{l}1 \\ 1\end{array}\right]$ is $k$-dimensional and the subspace of even functions is $\frac{k-1}{2}$-dimensional. Furthermore, each $k^{t h}$ order theta function has precisely $k$ zeros in any period parallelogram determined by $1, \tau$.

One of the first theorems [6] that one proves in this subject is that for $k \geq 3$, every even $k^{\text {th }}$ order theta function with characteristic $\left[\begin{array}{l}1 \\ 1\end{array}\right]$ vanishes at the three half periods

$$
\zeta=\frac{1}{2}, \frac{\tau}{2}, \frac{\tau+1}{2} .
$$

If we now restrict ourselves to the case $k=3$ we see immediately that

Theorem $1([6])$. For all $(\zeta, \tau) \in \mathbb{C} \times \mathbb{H}^{2}$,

$$
\begin{aligned}
& \theta\left[\begin{array}{c}
\frac{1}{3} \\
1
\end{array}\right](3 \zeta, 3 \tau)+\theta\left[\begin{array}{l}
\frac{1}{3} \\
1
\end{array}\right](-3 \zeta, 3 \tau) \\
& =c(\tau) \theta\left[\begin{array}{l}
0 \\
0
\end{array}\right](\zeta, \tau) \theta\left[\begin{array}{l}
0 \\
1
\end{array}\right](\zeta, \tau) \theta\left[\begin{array}{l}
1 \\
0
\end{array}\right](\zeta, \tau),
\end{aligned}
$$

where

$$
c(\tau)=\frac{2 \theta\left[\begin{array}{l}
\frac{1}{3} \\
1
\end{array}\right](0,3 \tau)}{\theta\left[\begin{array}{l}
0 \\
0
\end{array}\right](0, \tau) \theta\left[\begin{array}{l}
0 \\
1
\end{array}\right](0, \tau) \theta\left[\begin{array}{l}
1 \\
0
\end{array}\right](0, \tau)} .
$$

Set $x=\exp (\pi \imath \tau), z=\exp (2 \pi \imath \zeta)$ in the above theorem and get (for all $z \in \mathbb{C}$, $z \neq 0$, and all $x \in \mathbb{C},|x|<1)$

$$
\begin{gathered}
z \sum_{n=-\infty}^{\infty}(-1)^{n} x^{3 n^{2}+n} z^{3 n}+\sum_{n=-\infty}^{\infty}(-1)^{n} x^{3 n^{2}-n} z^{3 n} \\
=(1+z) \prod_{n=1}^{\infty}\left(1-x^{2 n}\right)\left(1-x^{4 n-2} z^{2}\right)\left(1-\frac{x^{4 n-2}}{z^{2}}\right)\left(1+x^{2 n} z\right)\left(1+\frac{x^{2 n}}{z}\right) .
\end{gathered}
$$

In order to get the usual version as in [1], for example, replace $x$ by $x^{\frac{1}{2}}$ and $z$ by $-z$.

The above proof connects QPI with a circle of ideas related to many other areas. It is another example, similar to the ones given in [4], where an interesting identity becomes immensely simplified when expressed in terms of theta functions with rational characteristics. 


\section{Computation}

In this section we recall the definitions and elementary properties of theta functions. We list those formulae needed to translate the last theorem to QPI.

Definition 1. The theta function with characteristic $\left[\begin{array}{c}\epsilon \\ \epsilon^{\prime}\end{array}\right] \in \mathbb{R}^{2}$ is defined on $\mathbb{C} \times \mathbb{H}^{2}\left(\mathbb{H}^{2}=\right.$ upper half plane $)$ by

$$
\theta\left[\begin{array}{c}
\epsilon \\
\epsilon^{\prime}
\end{array}\right](\zeta, \tau)=\sum_{n=-\infty}^{\infty} \exp 2 \pi \imath\left(\frac{1}{2}\left(n+\frac{\epsilon}{2}\right)^{2} \tau+\left(n+\frac{\epsilon}{2}\right)\left(\zeta+\frac{\epsilon^{\prime}}{2}\right)\right) .
$$

It is immediate that

$$
\theta\left[\begin{array}{c}
\epsilon \\
\epsilon^{\prime}
\end{array}\right](-\zeta, \tau)=\theta\left[\begin{array}{c}
-\epsilon \\
-\epsilon^{\prime}
\end{array}\right](\zeta, \tau)
$$

It is a consequence of the Jacobi triple product identity that with $x=\exp (\pi \imath \tau)$, $z=\exp (2 \pi \imath \zeta)$, we have

$$
\begin{aligned}
\theta\left[\begin{array}{c}
\epsilon \\
\epsilon^{\prime}
\end{array}\right](\zeta, \tau)= & \exp \left(\frac{\pi \imath \epsilon \epsilon^{\prime}}{2}\right) x^{\frac{\epsilon^{2}}{4}} z^{\frac{\epsilon}{2}} \prod_{n=1}^{\infty}\left(1-x^{2 n}\right)\left(1+\exp \left(\pi \imath \epsilon^{\prime}\right) x^{2 n-1+\epsilon} z\right) \\
& \times\left(1+\exp \left(-\pi \imath \epsilon^{\prime}\right) \frac{x^{2 n-1-\epsilon}}{z}\right) ;
\end{aligned}
$$

hence, in particular

$$
\begin{aligned}
& \theta\left[\begin{array}{l}
0 \\
0
\end{array}\right](\zeta, \tau)=\prod_{n=1}^{\infty}\left(1-x^{2 n}\right)\left(1+x^{2 n-1} z\right)\left(1+\frac{x^{2 n-1}}{z}\right), \\
& \theta\left[\begin{array}{l}
0 \\
1
\end{array}\right](\zeta, \tau)=\prod_{n=1}^{\infty}\left(1-x^{2 n}\right)\left(1-x^{2 n-1} z\right)\left(1-\frac{x^{2 n-1}}{z}\right), \\
& \theta\left[\begin{array}{l}
1 \\
0
\end{array}\right](\zeta, \tau)=x^{\frac{1}{4}} z^{\frac{1}{2}} \prod_{n=1}^{\infty}\left(1-x^{2 n}\right)\left(1+x^{2 n} z\right)\left(1+\frac{x^{2 n-2}}{z}\right)
\end{aligned}
$$

and

$$
\theta\left[\begin{array}{c}
\frac{1}{3} \\
1
\end{array}\right](0,3 \tau)=\left(\exp \frac{\pi \imath}{6}\right) x^{\frac{1}{12}} \prod_{n=1}^{\infty}\left(1-x^{2 n}\right) .
$$

As we saw, the infinite product (4) is single-valued for $\epsilon=0$; more generally, it is a single-valued function of $x^{\frac{1}{k}}$ for rational $\epsilon=\frac{m}{k}$ with $m \in \mathbb{Z}$.

From the definition (2) and elementary property (3) we see that

$$
\begin{gathered}
\theta\left[\begin{array}{c}
\frac{1}{3} \\
1
\end{array}\right](3 \zeta, 3 \tau)+\theta\left[\begin{array}{c}
\frac{1}{3} \\
1
\end{array}\right](-3 \zeta, 3 \tau) \\
=\frac{\left(\exp \frac{\pi \imath}{6}\right) x^{\frac{1}{12}}}{z^{\frac{1}{2}}}\left(z \sum_{n=-\infty}^{\infty}(-1)^{n} x^{3 n^{2}+n} z^{3 n}+\sum_{n=-\infty}^{\infty}(-1)^{n} x^{3 n^{2}-n} z^{3 n}\right) .
\end{gathered}
$$


On the other hand, the consequences of the Jacobi triple product identity listed above tell us that

$$
\begin{gathered}
c(\tau) \theta\left[\begin{array}{l}
0 \\
0
\end{array}\right](\zeta, \tau) \theta\left[\begin{array}{l}
0 \\
1
\end{array}\right](\zeta, \tau) \theta\left[\begin{array}{l}
1 \\
0
\end{array}\right](\zeta, \tau) \\
=\frac{\left(\exp \frac{\pi \imath}{6}\right) x^{\frac{1}{12}}}{x^{\frac{1}{4}} \prod_{n=1}^{\infty}\left(1-x^{2 n}\right)^{2}\left(1-x^{4 n-2}\right)^{2}\left(1+x^{2 n}\right)^{2}} \\
\times x^{\frac{1}{4}} z^{\frac{1}{2}}\left(1+\frac{1}{z}\right) \prod_{n=1}^{\infty}\left(1-x^{2 n}\right)^{3}\left(1-x^{4 n-2} z^{2}\right)\left(1-\frac{x^{4 n-2}}{z^{2}}\right)\left(1+x^{2 n} z\right)\left(1+\frac{x^{2 n}}{z}\right) .
\end{gathered}
$$

The equality of Theorem 1 therefore gives

$$
\begin{gathered}
z \sum_{n=-\infty}^{\infty}(-1)^{n} x^{3 n^{2}+n} z^{3 n}+\sum_{n=-\infty}^{\infty}(-1)^{n} x^{3 n^{2}-n} z^{3 n} \\
=\frac{(1+z) \prod_{n=1}^{\infty}\left(1-x^{2 n}\right)\left(1-x^{4 n-2} z^{2}\right)\left(1-\frac{x^{4 n-2}}{z^{2}}\right)\left(1+x^{2 n} z\right)\left(1+\frac{x^{2 n}}{z}\right)}{\prod_{n=1}^{\infty}\left(1-x^{4 n-2}\right)^{2}\left(1+x^{2 n}\right)^{2}}
\end{gathered}
$$

and since

$$
\prod_{n=1}^{\infty}\left(1-x^{4 n-2}\right)^{2}\left(1+x^{2 n}\right)^{2}=1
$$

we are done.

The proof of Theorem 1 in [6] is in fact quite easy. We show the points $z=\frac{1}{2}, \frac{\tau}{2}$ and $\frac{1+\tau}{2}$ are all zeros of the function defined by the left hand side of equation (1) and that this function has precisely three zeros (in a period parallelogram). In fact, the theorem contains more information and thus suggests that QPI can be generalized.

\section{Generalizations}

QPI proven in the previous section is the first in a long string of possible identities, each more complicated than its predecessor. Our proof was based on the study of even third order $\theta$-functions with characteristic $\left[\begin{array}{l}1 \\ 1\end{array}\right]$. We can use various other functions. Some will have interesting consequences.

3.1. It is rather easy to prove, using elliptic function theory, the following identity involving second order $\theta$-functions with characteristics $\left[\begin{array}{l}0 \\ 0\end{array}\right]$ and $\left[\begin{array}{l}0 \\ 1\end{array}\right]$ :

$$
\theta\left[\begin{array}{l}
0 \\
0
\end{array}\right](2 \zeta, 2 \tau)=c(\tau) \theta\left[\begin{array}{c}
0 \\
\frac{1}{2}
\end{array}\right](\zeta, \tau) \theta\left[\begin{array}{c}
0 \\
\frac{3}{2}
\end{array}\right](\zeta, \tau)
$$

where

$$
c(\tau)=\frac{\theta\left[\begin{array}{l}
0 \\
0
\end{array}\right](0,2 \tau)}{\theta\left[\begin{array}{c}
0 \\
\frac{1}{2}
\end{array}\right](0, \tau) \theta\left[\begin{array}{c}
0 \\
\frac{3}{2}
\end{array}\right](0, \tau)}
$$

and

$$
\theta\left[\begin{array}{l}
1 \\
1
\end{array}\right](2 \zeta, 2 \tau)=d(\tau) \theta\left[\begin{array}{l}
1 \\
1
\end{array}\right](\zeta, \tau) \theta\left[\begin{array}{l}
1 \\
0
\end{array}\right](\zeta, \tau)
$$


where

$$
d(\tau)=2 \frac{\theta^{\prime}\left[\begin{array}{l}
1 \\
1
\end{array}\right](0,2 \tau)}{\theta^{\prime}\left[\begin{array}{l}
1 \\
1
\end{array}\right](0, \tau) \theta\left[\begin{array}{l}
1 \\
0
\end{array}\right](0, \tau)} .
$$

The last two formulae are also immediate consequences of the Jacobi triple product identity which also tells us that

$$
c(\tau)=\prod_{n=1}^{\infty} \frac{1+x^{2 n}}{1-x^{2 n}}=d(\tau) .
$$

3.2. We describe in this subsection another identity which can be called the septagonal numbers identity. It is a consequence of the following result proven in [6].

Theorem 2. For all $(\zeta, \tau) \in \mathbb{C} \times \mathbb{H}^{2}$,

$$
\begin{aligned}
& \theta\left[\begin{array}{c}
\frac{3}{5} \\
1
\end{array}\right](0,5 \tau)\left(\theta\left[\begin{array}{c}
\frac{1}{5} \\
1
\end{array}\right](5 \zeta, 5 \tau)+\theta\left[\begin{array}{c}
-\frac{1}{5} \\
-1
\end{array}\right](5 \zeta, 5 \tau)\right) \\
- & \theta\left[\begin{array}{c}
\frac{1}{5} \\
1
\end{array}\right](0,5 \tau)\left(\theta\left[\begin{array}{c}
\frac{3}{5} \\
1
\end{array}\right](5 \zeta, 5 \tau)+\theta\left[\begin{array}{l}
-\frac{3}{5} \\
-1
\end{array}\right](5 \zeta, 5 \tau)\right) \\
= & c(\tau) \theta\left[\begin{array}{l}
0 \\
0
\end{array}\right](\zeta, \tau) \theta\left[\begin{array}{l}
0 \\
1
\end{array}\right](\zeta, \tau) \theta\left[\begin{array}{l}
1 \\
0
\end{array}\right](\zeta, \tau) \theta^{2}\left[\begin{array}{l}
1 \\
1
\end{array}\right](\zeta, \tau),
\end{aligned}
$$

where

$$
c(\tau)=\frac{a}{\theta\left[\begin{array}{l}
0 \\
0
\end{array}\right](0, \tau) \theta\left[\begin{array}{l}
0 \\
1
\end{array}\right](0, \tau) \theta\left[\begin{array}{l}
1 \\
0
\end{array}\right](0, \tau)}
$$

and $a \in \mathbb{C}, \neq 0$.

Substitutions similar to the ones used previously now yield

$$
\begin{gathered}
\sum_{n=-\infty}^{\infty}(-1)^{n} x^{5 n^{2}+n}\left(\sum_{n=-\infty}^{\infty}(-1)^{n} x^{5 n^{2}+3 n} z^{5 n+3}+\sum_{n=-\infty}^{\infty}(-1)^{n} x^{5 n^{2}-3 n} z^{5 n}\right) \\
-\sum_{n=-\infty}^{\infty}(-1)^{n} x^{5 n^{2}+3 n}\left(\sum_{n=-\infty}^{\infty}(-1)^{n} x^{5 n^{2}+n} z^{5 n+2}+\sum_{n=-\infty}^{\infty}(-1)^{n} x^{5 n^{2}-n} z^{5 n+1}\right) \\
=(1+z)(1-z)^{2} \prod_{n=1}^{\infty}\left(1-x^{2 n}\right)^{2}\left(1-x^{2 n} z\right)\left(1-\frac{x^{2 n}}{z}\right)\left(1-x^{4 n-2} z^{2}\right)\left(1-\frac{x^{4 n-2}}{z^{2}}\right) \\
\times\left(1-x^{4 n} z^{2}\right)\left(1-\frac{x^{4 n}}{z^{2}}\right) .
\end{gathered}
$$


3.3. We proceed to explain the role of $k^{t h}$ order $\theta$-functions in this work. For odd $k \geq 3$, every nontrivial element in the $\frac{k-1}{2}$-dimensional vector space $\mathcal{E}_{k}\left[\begin{array}{l}1 \\ 1\end{array}\right]$ of even $k^{\text {th }}$ order $\theta$-functions with characteristic $\left[\begin{array}{l}1 \\ 1\end{array}\right]$ vanishes at the three half periods $\frac{1}{2}, \frac{\tau}{2}, \frac{1+\tau}{2}$ and at $k-3$ additional points. A function

$$
f(\zeta, \tau)=\theta\left[\begin{array}{l}
0 \\
0
\end{array}\right](\zeta, \tau) \theta\left[\begin{array}{l}
0 \\
1
\end{array}\right](\zeta, \tau) \theta\left[\begin{array}{l}
1 \\
0
\end{array}\right](\zeta, \tau) \prod_{i=1}^{k-3} \theta\left[\begin{array}{l}
\epsilon_{i} \\
\epsilon_{i}^{\prime}
\end{array}\right](\zeta, \tau)
$$

belongs to $\mathcal{E}_{k}\left[\begin{array}{l}1 \\ 1\end{array}\right]$ if and only if (the additional zeros of this function are at $\left.\frac{1-\epsilon_{i}^{\prime}}{2}+\frac{1-\epsilon_{i}}{2} \tau\right)$

$$
\sum_{i=1}^{k-3} \epsilon_{i} \equiv \sum_{i=1}^{k-3} \epsilon_{i}^{\prime} \equiv 0 \quad \bmod 2
$$

and for each integer $i, 0 \leq i \leq k-3$, there exists an integer $i^{\prime}, 0 \leq i^{\prime} \leq k-3, i \neq i^{\prime}$, such that

$$
\left[\begin{array}{c}
\epsilon_{i} \\
\epsilon_{i}^{\prime}
\end{array}\right] \equiv-\left[\begin{array}{c}
\epsilon_{i^{\prime}} \\
\epsilon_{i^{\prime}}^{\prime}
\end{array}\right] \bmod 2
$$

(in particular, for $\left.\epsilon_{i}=1=\epsilon_{i}^{\prime}\right)^{2}$.

The $\frac{k-1}{2}$ functions

$$
\varphi_{l}(\zeta, \tau)=\frac{\theta\left[\begin{array}{c}
\frac{2 l+1}{k} \\
1
\end{array}\right](k \zeta, k \tau)+\theta\left[\begin{array}{c}
\frac{2 l+1}{k} \\
1
\end{array}\right](-k \zeta, k \tau)}{2 \theta\left[\begin{array}{c}
\frac{2 l+1}{k} \\
1
\end{array}\right](0, k \tau)}, l=0,1, \ldots, \frac{k-3}{2},
$$

form a basis for $\mathcal{E}_{k}\left[\begin{array}{l}1 \\ 1\end{array}\right]$. We have normalized these basis functions to assume the value 1 at $\zeta=0$; their Taylor expansions at the origin are

$$
\varphi_{l}(\zeta, \tau)=1+\sum_{i=1}^{\infty} \alpha_{l i} \zeta^{i}
$$

There exist constants $c_{j}(\tau)$ such that

$$
f(\zeta, \tau)=\sum_{l=0}^{\frac{k-3}{2}} c_{l}(\tau) \varphi_{l}(\zeta, \tau)
$$

Rewriting these identities in terms of appropriate local coordinates ( $x$ and $z$ ) leads to product formulae.

QPI is the case $k=3$. The next case of interest is $k=5$. For this $k$, it involves no loss of generality to take

$$
\epsilon_{1}=\epsilon, 0 \leq \epsilon<2, \epsilon_{1}^{\prime}=\epsilon^{\prime}, 0 \leq \epsilon^{\prime}<2,
$$

which implies that

$$
\epsilon_{2}=2-\epsilon, \epsilon_{2}^{\prime}=2-\epsilon^{\prime}
$$

\footnotetext{
${ }^{2}$ These necessary and sufficient conditions are a translation of the fact that in an $N$-dimensional vector space of analytic functions, we can always find a function that vanishes at a prescribed set of $N-1$ points.
} 
(these conditions also suffice). The septagonal numbers identity is obtained by studying the case $\epsilon=1=\epsilon^{\prime}$. The cases $\epsilon=1, \epsilon^{\prime}=0$ and $\epsilon=0, \epsilon^{\prime}=1$ should also be interesting.

3.4. Among the consequences of QPI are the following two identities for functions closely related to the Ramanujan partition functions $P_{N}$ studied in our paper [7]:

$$
\sum_{n=-\infty}^{\infty}(6 n+1) x^{\frac{3 n^{2}+n}{2}}=\prod_{n=1}^{\infty}\left(1-x^{n}\right)^{3}\left(1-x^{2 n-1}\right)^{2}
$$

and

$$
\sum_{n=-\infty}^{\infty}(-1)^{n}(3 n+2) x^{3 n^{2}+4 n+1}=\prod_{n=1}^{\infty}\left(1-x^{2 n}\right)^{3}\left(1+x^{n}\right)^{2}=\prod_{n=1}^{\infty} \frac{\left(1-x^{2 n}\right)^{3}}{\left(1-x^{2 n-1}\right)^{2}} .
$$

The first of these identities has the following combinatorial interpretation. Partition the positive integers using three copies of the even integers and five copies of the odd integers. Let $E(n)$ (respectively, $O(n)$ ) be the number of even (odd) such partitions of $n \in \mathbb{Z}^{+}$. Then

$$
E(n)-O(n)=\left\{\begin{array}{r}
0 \text { if } n \neq \frac{3 m^{2}+m}{2} \text { for all } m \in \mathbb{Z}, \\
(6 m+1) \text { if } n=\frac{3 m^{2}+m}{2} \text { for } m \in \mathbb{Z} .
\end{array}\right.
$$

The combinatorial interpretation of the equality of the first and third terms of the (it is obvious that the second and third terms are equal) second identity is a bit more complicated. Our partitions now use three copies of the even integers, each one with a different color, and we allow repetitions of the odd integers although we still think of the odd integers with two colors. As in the previous situation we denote by $E(n)$ and $O(n)$ the number of even and odd partitions, respectively. The definition of even partition now is that there are an even number of even integers in the partition. In this case the difference $E(n)-O(n)$ is zero unless $n$ is of the form $3 m^{2}+4 m+1$. In the latter case the difference is $(-1)^{m}(3 m+2)$. As usual, the first equality of the second identity gives us an alternate combinatorial interpretation. We use three primary colors of even integers and two secondary colors of all the integers for our partitions, without allowing repetitions. We consider a partition to be even if we use an even number of (even) integers of primary colors.

The proof of the identities is based on Theorem 1. We differentiate the identity of that theorem and set $\zeta=\frac{1}{2}$. This yields

$$
\begin{aligned}
& 3 \theta^{\prime}\left[\begin{array}{c}
\frac{1}{3} \\
1
\end{array}\right]\left(\frac{3}{2}, 3 \tau\right)-3 \theta^{\prime}\left[\begin{array}{c}
\frac{1}{3} \\
1
\end{array}\right]\left(-\frac{3}{2}, 3 \tau\right) \\
& =c(\tau) \theta^{\prime}\left[\begin{array}{l}
1 \\
0
\end{array}\right]\left(\frac{1}{2}, \tau\right) \theta\left[\begin{array}{c}
0 \\
0
\end{array}\right]\left(\frac{1}{2}, \tau\right) \theta\left[\begin{array}{l}
0 \\
1
\end{array}\right]\left(\frac{1}{2}, \tau\right) .
\end{aligned}
$$

If we now use the definition of $c(\tau)$ given in Theorem 1 and the most elementary properties of theta functions, we rewrite the last equation as

$$
3\left(\exp \left(\frac{2 \pi \imath}{3}\right)-\exp \left(-\frac{\pi \imath}{3}\right)\right) \theta^{\prime}\left[\begin{array}{c}
\frac{1}{3} \\
0
\end{array}\right](0,3 \tau)=2 \frac{\theta\left[\begin{array}{l}
\frac{1}{3} \\
1
\end{array}\right](0,3 \tau) \theta^{\prime}\left[\begin{array}{l}
1 \\
1
\end{array}\right](0, \tau)}{\theta\left[\begin{array}{l}
1 \\
0
\end{array}\right](0, \tau)}
$$


Use of Jacobi's identity and elementary manipulations replaces the last equality by

$$
-3 \exp \left(\frac{2 \pi \imath}{3}\right) \theta^{\prime}\left[\begin{array}{l}
\frac{1}{3} \\
0
\end{array}\right](0,3 \tau)=\pi \theta\left[\begin{array}{l}
\frac{1}{3} \\
1
\end{array}\right](0,3 \tau) \theta\left[\begin{array}{l}
0 \\
0
\end{array}\right](0, \tau) \theta\left[\begin{array}{l}
0 \\
1
\end{array}\right](0, \tau) .
$$

It thus follows that

$$
-3 \exp \left(\frac{2 \pi \imath}{3}\right) \theta^{\prime}\left[\begin{array}{c}
\frac{1}{3} \\
0
\end{array}\right](0,3 \tau)=\pi \exp \left(\frac{\pi \imath}{6}\right) x^{\frac{1}{12}} \prod_{n=1}^{\infty}\left(1-x^{2 n}\right)^{3}\left(1-x^{4 n-2}\right)^{2} .
$$

All that remains in order to obtain the formula we seek is the expansion of the left hand side as a power series in $x=\exp (\pi \imath \tau)$. Since

$$
\begin{aligned}
& \theta^{\prime}\left[\begin{array}{c}
\frac{1}{3} \\
0
\end{array}\right](0,3 \tau)=\sum_{n=-\infty}^{\infty} 2 \pi \imath\left(n+\frac{1}{6}\right) \exp \left(2 \pi \imath\left(\frac{1}{2}\left(n+\frac{1}{6}\right)^{2} 3 \tau\right)\right. \\
& =\frac{\pi \imath}{3} x^{\frac{1}{12}} \sum_{n=-\infty}^{\infty}(6 n+1) x^{3 n^{2}+n},
\end{aligned}
$$

the final identity is

$$
\sum_{n=-\infty}^{\infty}(6 n+1) x^{3 n^{2}+n}=\prod_{n=1}^{\infty}\left(1-x^{2 n}\right)^{3}\left(1-x^{4 n-2}\right)^{2},
$$

which after the obvious change of variables becomes (5).

The second identity is obtained in a similar fashion by evaluating the identity in Theorem 1 at $\zeta=\frac{\tau}{2}$ instead of $\zeta=\frac{1}{2}$. In fact it is possible to obtain a third identity in this way by evaluating at $\zeta=\frac{1+\tau}{2}$.

\section{REFERENCES}

1. K. Alladi, The quintuple product identity and shifted partition functions, Comput. Math. Appl. 68 (1996), 3-13. MR 98c:05012

2. R. Brooks, H.M. Farkas, and I. Kra, Number theory, theta identities and modular curves, Contemporary Math. 201 (1997), 125-154. CMP 97:07

3. L. Carlitz and M.V. Subbarao, A simple proof of the quintuple product identity, Proc. Amer. Math. Soc. 32 (1972), 42-44. MR 44:6507

4. H.M. Farkas and Y. Kopeliovich, New theta constant identities II, Proc. Amer. Math. Soc. 123 (1995), 1009-1020. MR 95e:11050

5. H.M. Farkas, Y. Kopeliovich, and I. Kra, Uniformization of modular curves, Comm. Anal. Geom. 4 (1996), 207-259. MR 97j:11019a

6. H.M. Farkas and I. Kra, Theta constants, Riemann surfaces and the modular group, in preparation.

7. _ A function theoretic approach to the Ramanujan partition identities with applications to combinatorial number theory, Proc. of Iberoamerican Congress on Geometry, Chile 1998, pp. $75-106$.

8. B. Gordon, Some identities in combinatorial analysis, Quart. J. Math. Oxford 12 (1961), 285-290. MR 25:21

9. G.N. Watson, Theorems stated by Ramanujan, (VII): Theorems on continued fractions, J. London Math.Soc. 4 (1929), 39-48.

Department of Mathematics, The Hebrew University of Jerusalem, Jerusalem 91904, ISRAEL

E-mail address: farkas@math.huji.ac.il

Department of Mathematics, State University of New York at Stony Brook, Stony Brook, New York 11794

E-mail address: irwin@math.sunysb.edu 